\title{
Extradermal melanin transfer? Lack of macroscopic spleen melanization in old C57BL/6 mice with de-synchronized hair cycle ${ }^{\star}$
}

\author{
Dominika Michalczyk, Malgorzata Popik, Aleksander Salwinski and \\ Przemyslaw M. Plonka ${ }^{\bowtie}$ \\ Department of Biophysics, Faculty of Biochemistry, Biophysics and Biotechnology, Jagiellonian University, \\ Kraków, Poland
}

Received: 07 April, 2009; revised: 04 June, 2009; accepted: 17 June, 2009

available on-line: 18 June, 2009

\begin{abstract}
In quest of alternate, extradermal path of melanin transfer from skin to the visceral organs, we suggested that some portions of such melanin may be deposited in the spleen, which in young black C57BL/6 mice is often melanized. Here, we confirm these observation using young C57BL/6 female mice (up to 17 weeks) and show that this phenomenon cannot be observed in old animals where the hair cycle is not synchronized any more. The experiments were carried out both on spontaneous and depilation-induced hair cycle. We have checked it as a side-observation over many other experiments carried out on young and old C57BL/6 female mice (up to 2.5 years of life). The presence or absence of melanin in the spleens was checked macroscopically, and histologically by Fontana-Masson (FM) staining, and synchronization of the hair cycle - by standard histomorphometric analysis of the back skin hair follicles. In about $40 \%$ of old spleens black FM-stainable "debris" could be found under closer histological examination. This study shows that, at least in part, the phenomenon of splenic melanosis in C57BL/6 mice can be correlated with the synchronized skin melanization parallel to the hair cycle progress, and that splenic melanin undergoes gradual degradation during the mouse life.
\end{abstract}

Keywords: ageing, Fontana-Masson staining, hair cycle, lipofuscin, melanin degradation, spleen melanosis

\section{INTRODUCTION}

The pigmentation synapse is the fourth known type of synapses besides neurological, phagocytic (engulfment) and immunological ones (Van den Bossche et al., 2006). It enables melanocytes to transfer melanin to the target epidermal or hair follicle keratinocytes, which indicates the relation of this process to the better known processes of exo-, endoand, perhaps, autophagocytosis (Schraermeyer, 1991;
Izumi et al., 2003; Palumbo, 2003; Van den Bossche et al., 2006). However, there may exist an alternate, extradermal path for melanin transfer to the visceral organs (Wasserman, 1967), e.g. the spleen, which in black mice can reveal melanosis (Weissman, 1967). On the other hand, there is a strong correlation between the activity of the immunological system and the hair follicular system: the proliferatory activity of splenocytes depends on the phase of the hair cycle (Slominski et al., 1997), and the hair cycle, includ-

\footnotetext{
$\square$ Corresponding author: Przemyslaw M. Plonka, Department of Biophysics, Faculty of Biochemistry, Biophysics and Biotechnology, Jagiellonian University, Gronostajowa 7, 30-387 Kraków, Poland; tel.: (48) 12664 6350; fax: (48) 12 664 6902; e-mail: przemyslaw.plonka@uj.edu.pl,mieszkoy@yahoo.com

^ Preliminary report of this work was presented by D.M. at the XXXVI Winter School "Molecule interactions in health and disease" organized by the Faculty of Biochemistry, Biophysics and Biotechnology, Jagiellonian University, 21-26 February, 2009, Zakopane, Poland.

Abbreviations: C57BL/6, Cross 57th-generation female $\times 52$ nd-genertion male Black, 6th sub-strain (inbred mouse strain); CYP, cyclophosphamide, EPR, electron paramagnetic resonance; FM, Fontana-Masson staining; H \& E, haematoxylin and eosin staining; MNA, 1-methylnicotinamide; NIC, Nomarski interference contrast; NOS 1, nitric oxide synthase 1 (neuronal); NOS 3, nitric oxide synthase 3 (endothelial); PBS, phosphate-buffered saline.
} 
ing its melanogenic aspect (Slominski et al., 1991), is regulated by immunological mechanisms (Paus et al., 1999; Stenn \& Paus, 2001; Schneider et al., 2009).

The phenomenon of splenic melanosis in young C57BL/6 animals has been known for over 40 years (Weissman, 1967), but its biochemical, physiological and genetic basis remains poorly understood. The black pigment was found mainly in the extracellular space of the red pulp or in macrophages (Veninga et al., 1989), in the dorso-cranial part (anterior pole) (Van der Heijden et al., 1995) or the posterolateral aspects of the spleen (Weissman, 1967). Some authors identify the melanin-bearing splenic cells as "melanophores" (Van der Heijden et al., 1995) akin to melanocytes. The occurence of splenic melanosis varies between particular reports $(10-21 \%$, Weissman, 1967; 8-34\%, Crichton et al., 1978; 15\%, Veninga et al., 1989; 23\%, van der Heijden et al., 1995; 14-80\%, Plonka et al., 2005b) but rarely exceeds 30\% (34\% for female C57BL/6J and 31\% for female C57BL/IOScSn, Crichton et al., 1978; 50-80\% for female C57BL/6 in late catagen/early telogen, Plonka et al., 2005b). As the reason of this pigmentatory effect some authors suggested haemosiderins (Veninga et al., 1989) or lipofuscin (Crichton et al., 1978), but most of them, starting with Weissman (1967) and followed by Sundberg (1991), van der Heijden et al. (1995), and recently by us (Plonka et al., 2005b), insist that melanin is the pigment responsible for the phenomenon.

A few years ago we confirmed by electron paramagnetic resonance (EPR) spectroscopy that the splenic pigment is of the eumelanin type (Plonka et al., 2005b). We also found a correlation between the occurrence of the phenomenon and the hair cycle, and reported the presence of as many as $50-80 \%$ melanotic spleens in mice in late catagen (the involution phase of the hair cycle) or early telogen (the "resting" phase in the hair cycle), i.e. very shortly after macroscopically appreciable anagen (the growth phase of the hair cycle), while in mice in deep telogen the percentage of melanotic spleens was about 15\% (Plonka et al., 2005b).

Splenic eumelanin observed by EPR differed from the pigment found in the skin (follicular melanocytes and hair shafts). Its power saturability and line width betrayed heterogeneity of paramagnetic centres as compared to the normal skin pigment, which suggested a partial degradation or other kind of metamorphosis of the pigment (Plonka et al., 2005b). At the same time we confirmed a stochastic and a yes-or-no character of the phenomenon which means that without laparotomy only the probability that a spleen reveals melanosis can be determined, and no gradual change in the saturation of the black (from light gray to deep black) but instead a variability in the area of the black spots can be observed. In neither of the cited papers it was clarified wheth- er melanin is produced in spleens de novo, whether this production is synchronized with any physiological signal of a systemic range which might at the same time control hair cycling, or whether melanin is transferred from other sites of the organism (perhaps from skin with hair follicles) to be stored in the spleen.

Most of the cited authors together with us in our early study dealt with young mice up to 10-15 weeks after birth, and so far no study of old animals (over 1.5 year) has been performed. It is of great interest to check whether the phenomenon of splenic melanosis persists throughout the mouse life and whether it is somehow correlated with consecutive hair cycles. The fate of melanotic spots observed in the spleens of young animals is also very intriguing - does the melanosis remain to the end of the animal's life? If so, the fraction of pigmented spleens in old animals should be similar to that in young animals. And if the spleen is a place of storage of melanin coming from hair follicles in consecutive anagens - the fraction of melanotic spleens should even increase over the life period.

However, from the EPR properties of the pigment (Plonka et al., 2005b) we expect a gradual degradation of the splenic melanin. Starting with 3rd hair cycle after birth, transformation of hair follicles according to subsequent stages of the hair cycle becomes asynchronous (Dry, 1926; Chase \& Eaton, 1959; Militzer, 2001; Young et al., 2003; Plikus \& Choung, 2004; 2008). If the phenomenon of splenic melanosis remains somehow related to hair cycling, then de-synchronization of hair follicles in old animals should be associated with less pronounced splenic melanosis. However, if the spleen serves as a place of storage, but not degradation of melanin, it should remain pigmented, or even the pigmentation should increase during the lifetime. As synchronized hair cycling co-exists with spleen melanosis in young C57BL/6 mice, we presume that in the case of a de-synchronized hair cycle in very old animals the presence or absence of spleen melanization will be a valuable clue concerning the origin and fate of splenic melanin and potential cross-talk between hair cycling and splenic melanosis.

\section{MATERIALS AND METHODS}

Animals. Young female C57BL/6 mice (about 6-7 weeks old) were purchased from the Animal Breeding Facility of the Silesian Medical Academy in Katowice - Ligota (Poland). Nos3 knockout mice in C57BL/6 background were a generous gift from Professor Stefan Chlopicki (Jagiellonian University Medical College). Several groups of mice (for details see Tables 1 and 2) were kept from 7 to 133 
Table 1. Spleen melanosis in young (up to 9 months) female C57BL/6 mice in control groups of various experiments.

\begin{tabular}{|c|c|c|c|c|c|}
\hline Age (weeks) & Genotype & $\begin{array}{c}\text { Total number of } \\
\text { spleens }\end{array}$ & $\begin{array}{c}\text { Number of } \\
\text { pigmented spleens }\end{array}$ & $\begin{array}{c}\text { Pigmented spleens } \\
(\%)\end{array}$ & $\begin{array}{c}\text { Average pigmented area } \\
(\%)\end{array}$ \\
\hline $9^{*}$ & $w t$ & 11 & 4 & 36.4 & 41.3 \\
\hline $8-11^{*}$ & $\mathrm{Nos}^{-/-}$ & 14 & 5 & 35.7 & 24.5 \\
\hline $10^{*}$ & $w t$ & 49 & 23 & 46.9 & 41.7 \\
\hline $11^{*}$ & $w t$ & 9 & 2 & 22.2 & 20 \\
\hline $12^{*}$ & $w t$ & 20 & 4 & 20 & 20.6 \\
\hline $13^{*}$ & $w t$ & 10 & 5 & 50 & 20.8 \\
\hline $16^{*}$ & $w t$ & 1 & 0 & 0 & 0 \\
\hline 17 & $w t$ & 7 & 2 & 28.6 & 40 \\
\hline $36^{*}$ & $\mathrm{Nos}^{-/-}$ & 3 & 0 & 0 & 0 \\
\hline Total & & 124 & 45 & 36.29 & \\
\hline Mean & & & & 25.51 & 23.05 \\
\hline \pm S.D. & & & & 19 & 17.12 \\
\hline
\end{tabular}

Nos3 $^{-1}$, Nos3 knockout mice; *depilated 7-8 weeks after birth.

weeks, i.e. about 2.5 years, over a 4 -year period of activity of the Trichology Group at the Department of Biophysics, Jagiellonian University, and served to execute observations described here as side-observations made during other on-going experiments. The animals were kept in the animal facility, under standard conditions, in community cages and a 12$\mathrm{h}$ day/night regime, with continuous access to food and drinking water. They were monitored for any dermatological changes which were photodocumented. Permission to carry out the animal study was granted by the 1st Local Committee for Animal Research in Kraków (15/OP/2004).

Hair cycle control. To achieve a perfectly synchronized anagen development over the whole back skin area, hair shafts of the back fur coat of mice with all follicles in telogen (pink skin) were depilated using a warm mixture $(1: 1)$ of beeswax (Al- drich, Milwaukee, WI, USA) and gum rosin (Sigma, St Louis, MO, USA) according to Paus et al. (1990). The procedure was done on young animals (7-8 weeks) in deep ketamine anaesthesia (Ketanest 50; Parke-Davis GmbH, Berlin, Germany; i.p. $0.75 \mathrm{mg} /$ mouse, in PBS). To obtain skin in synchronized catagen animals were treated with topical dexamethasone $(0.8-1 \mathrm{ml}$ dexamethasone-21-acetate in propylene glycol; Sigma, St Louis, MO, USA) since day 10 after depilation (Paus et al., 1994). 1-Methylnicotinamide (MNA, chlorate; $15 \mathrm{mg} / 100 \mathrm{ml}$ drinking water; Dr. Jan Adamus, Institute of Applied Radiation Chemistry, Technical University, Łódź, Poland) was administered continuously starting with week 30 after birth. To disrupt normal hair cycle, cyclophosphamide (CYP; Endoxan, ASTA Medica AG, Frankfurt, Germany; in saline or in PBS) was given in a single dose of 120 or $150 \mathrm{mg} / \mathrm{kg}$ body mass i.p. on

Table 2. Lack of splenic melanosis of various treated and untreated groups of old (over 1 year) female C57BL/6 mice.

\begin{tabular}{|c|c|c|c|c|}
\hline Treatment & Genotype & Age (weeks) & $\begin{array}{c}\text { Total number of } \\
\text { spleens }\end{array}$ & $\begin{array}{c}\text { Number of pigmented } \\
\text { spleens }\end{array}$ \\
\hline Untreated & $w t$ & 60 & 2 & 0 \\
\hline untreated & $\mathrm{Nos}^{-/-}$ & 60 & 3 & 0 \\
\hline MNA & $\mathrm{Nos}^{-/-}$ & 60 & 4 & 0 \\
\hline MNA & $w t$ & 60 & 4 & 0 \\
\hline untreated & $\mathrm{Nos}^{-/-}$ & 77 & 6 & 0 \\
\hline CYP $120 \mathrm{mg} / \mathrm{kg}^{*}$ & $w t$ & 83 & 1 & 0 \\
\hline CYP $120 \mathrm{mg} / \mathrm{kg}^{*}$ & $w t$ & 87 & 5 & 0 \\
\hline CYP $150 \mathrm{mg} / \mathrm{kg}^{*}$ & $w t$ & 87 & 6 & 0 \\
\hline untreated ${ }^{*}$ & $w t$ & 114 & 1 & 0 \\
\hline MNA* & $w t$ & 133 & 2 & 0 \\
\hline CYP $150 \mathrm{mg} / \mathrm{kg}^{*}$ & $w t$ & 133 & 1 & 0 \\
\hline CYP $150 \mathrm{mg} / \mathrm{kg}, \mathrm{MNA}^{*}$ & $w t$ & 133 & 2 & 0 \\
\hline Total & & & 37 & 0 \\
\hline Total untreated & & & 13 & 0 \\
\hline
\end{tabular}

Nos3 $^{-/-}$, Nos3 knockout mice; MNA, 1-methylnicotinamide $15 \mathrm{mg} / 100 \mathrm{ml}$ in drinking water; CYP, cyclophosphamide; *depilated 7-8 weeks after birth. 
day 9 after depilation (Paus et al., 1994; Hendrix et al., 2005).

Macroscopic observations and photodocumentation. The presence and area of spleen melanosis was estimated shortly after animal sacrifice, during necropsy. After fixation in formalin pictures of spleens and skin specimens were taken using Nikon Digital Camera D80 with Af-S Nikkor 18-70 mm 1:3.5-4.5G ED lenses or an analog Pentax camera adjusted for macrophotography.

Because of the de-synchronization of hair growth in old animals, only the percentage of the back skin area between anagen IIIc-catagen V could be judged by the size and number of black spots indicating active melanogenesis and the presence of melanin granules in the region of hair follicle or in the hair shaft above the keratogenous zone (MuellerRoever et al., 2001). It was checked after skin fixation.

Preparation of skin and spleen sections. Animals were sacrificed by cervical dislocation after shawing (Braun animal shaver) in deep ketamine anaesthesia (see, Hair cycle control). Skin was separated from the body on the level of the subcutis by scissors, and spread on a piece of cardboard, whereupon spleen was removed, and both organs immediately fixed in buffered $(\mathrm{pH}$ 7.4) 5\% formalin (POCh, Gliwice, Poland) (Paus et al., 1990).

For histology, formalin-fixed skin samples of the middle regions of the back, and spleens were dehydrated, embedded in paraffin (Histoplast, Thermo Shandon, Runcorn, UK) and stained by haematoxylin (Mayer haematoxylin, Stamar, Dąbrowa Górnicza, Poland) and eosin (POCh, Gliwice, Poland) according to Romeis (1991) and Shirai et al. (2001) (skin) or by sodium thiosulphate and silver nitrate $(\mathrm{V})$, followed by neutral red (all: POCh, Gliwice, Poland) according to Fontana (1912), Masson (1914) and Bancroft \& Stevens (1982) (Fontana-Masson (FM) staining of spleens). The tissues were sliced using a manual microtome Finesse 325 (Thermo Shandon, Runcorn, UK) with MX35 Plus Premier (Thermo Electron Corporation, USA) knife and embedded using Consult Mount mounting medium (Thermo Shandon, Pittsburgh, USA). The thickness of slices varied between 8-10 $\mu \mathrm{m}$ for skin and 1-3 $\mu \mathrm{m}$ for spleen.

Representative sections were used for photodocumentation with microscopes Elipse TS 100 (Nikon Corporation, Tokyo, Japan) (magnification $2 \times, 10 \times, 20 \times$ ) equipped with a digital camera SSCDC 54AP (Sony, Japan), and Leica DM IRE2 (Leica Microsystems, Wetzlar, Germany) with Nomarski interference contrast (NIC), equipped with a digital camera DC 350FX (magnification 100×, Leica Microsystems, Wetzlar, Germany).
Statistical analysis. Macroscopic observations of spleens were pooled for every independent experiment, and means \pm S.D. were calculated both for the percentage of melanotic spleens and for the areas of macroscopic melanosis. Histomorphometry was executed according to Mueller-Roever et al. (2001) evaluating 20-100 hair follicles per skin area per mouse and calculating the percentage of hair follicles in particular hair cycle stages. The data are shown for selected animals without pooling.

\section{RESULTS AND DISCUSSION}

Our results indicate that in C57BL/6 mice a cross-talk exists between mechanisms of synchronized hair cycling and splenic melanosis, suggesting that this organ is a site of transient storage and degradation of the pigment. It can be concluded from the following observations:

1. Splenic melanosis is present in young animals with synchronized hair cycle and the percentage of melanotic spleens correlates with the progress of the hair cycle (Plonka et al., 2005b),

2. Macroscopic splenic melanosis is absent in old C57BL/6 mice with de-synchronization of the hair cycle,

3. Microscopically appreciable FM-stainable "melanin debris" is present in some old, macroscopically non-pigmented spleens, and in pigmented spleens in their non-pigmented parts, but not in young non-pigmented spleens.

\section{Splenic melanosis in young C57BL/6 animals with synchronized hair cycling}

As it can be seen in Fig. 1, it is easy to synchronize hair cycling in the whole back skin of young C57BL/6 mice by depilation in telogen of the second postnatal hair cycle (Paus et al., 1990; Mueller-Roever et al., 2001). The mice reveal a uniform pink colour of back skin which changes in parallel to the progress of the hair cycle. Similarly, pre-mature but synchronous catagen can be induced by topical dexamethasone when applied since day 10 p.d. (Paus et al., 1994). The synchronization of hair cycling can be appreciated on macroscopic pictures (Fig. 1A-C) where the back skin colouration has been revealed by skin depilation, on histological skin sections (Fig. 1D-F) with hair follicles representing morphology typical of a given hair cycle stage (Mueller-Roever et al., 2001), and on histomorphometry histograms (Fig. 2) where hair follicles in particular stages present clearly distinguishable groups. In such animals, spleens may reveal melanosis (Fig. 2B and C, Ta- 


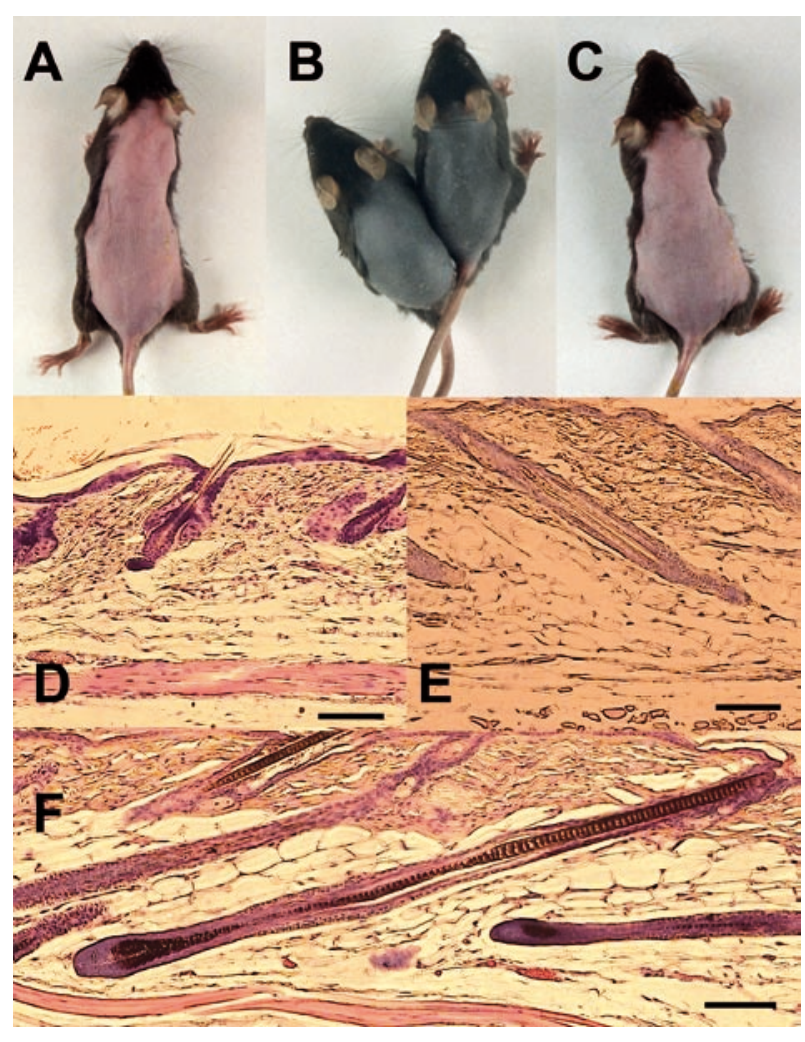

Figure 1. Synchronization of the hair cycle in young (7-9 weeks old) female $\mathrm{C} 57 \mathrm{Bl} / 6$ mice.

Macroscopical pictures (A-C), and corresponding histological skin sections (D-F, H \& E staining). Telogen (A, D), early anagen VI $(\mathrm{B}, \mathrm{F})$ and even catagen $(\mathrm{C}, \mathrm{E})$ skin can be easily distinguished by the difference in shade, which illustrates high synchronization of the hair cycle. In the case of $\mathrm{C}$ - dexamethasone-induced pre-mature catagen skin is shown, to obtain a more uniform gray skin shade associated with this hair cycle stage, than in the case of a spontaneous catagen. The skin was depilated again to display its gray shade. Note the difference in skin thickness. Scale bars, $0.1 \mathrm{~mm}$.

ble 1) in the dorso-cranial part (Fig. 2B), but sometimes even in the middle, in the form of a black spot (Fig. 2C). Besides melanotic ones, spleens of a normal phenotype devoid of black pigmentation can be found in all the experimental groups (Fig. 2A, Table 1).

These observation can serve as a supplement to our previous report where we studied the C57BL/6 mice spleens with EPR spectroscopy and suggested that the splenic melanosis might be related to the hair follicular melanogenesis (Plonka et al., 2005b). It must be emphasized that in this model (Paus et al., 1990; Slominski \& Paus, 1993; Slominski et al., 1991; 1994; 2004) all truncal skin melanocytes are confined to hair follicles and no melanocytes are present in the truncal skin epidermis of mature C57BL/6 mice (Chase, 1954). If, therefore, their splenic melanosis is really correlated with skin melanin production, such correlation must au-

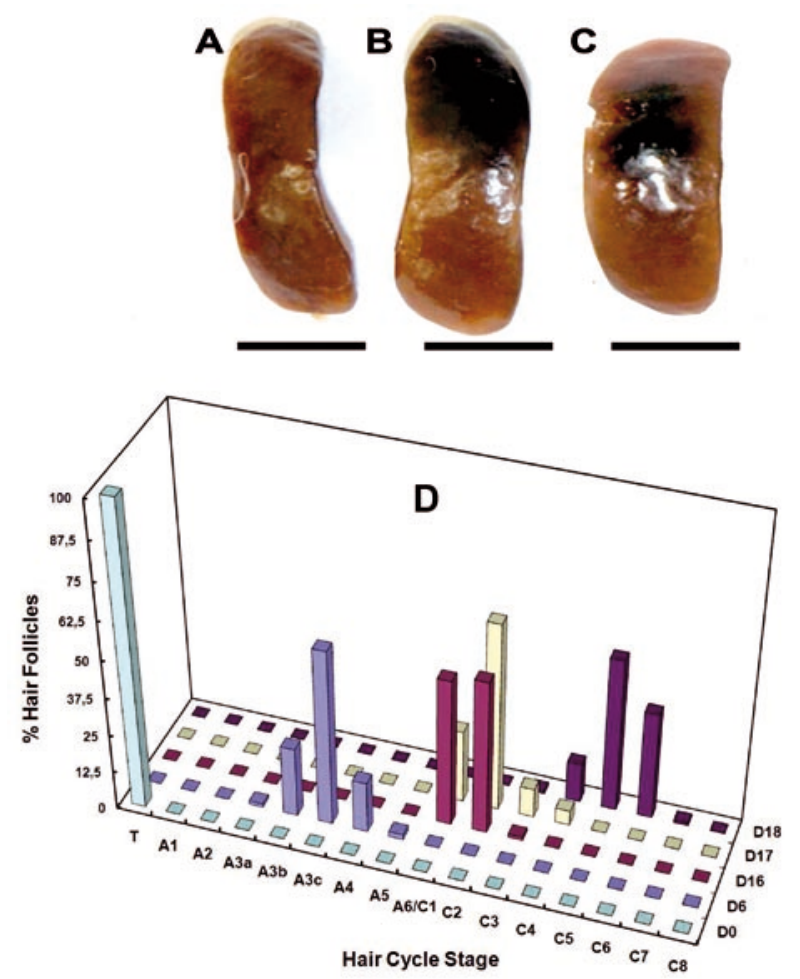

Figure 2. Association of macroscopic splenic melanosis with synchronous hair cycling in young C57BL/6 mice. The phenomenon appears in a stochastic mode - besides normally pigmented (A), some spleens reveal melanosis $(\mathrm{B}, \mathrm{C}) . \mathrm{C}$, atypical melanosis as a spot in the middle of the dorsal part of the spleen. Scale bars, $5 \mathrm{~mm}$. D, histomorphometry of hair follicles in the middle part of the back skin of young mice. D0-D18, day after anagen induction. A1-A6, C1-C8, T, stages (uppercase letters) and sub-stages (Arabic numbers and lowercase letters) of hair cycle. A, anagen; C, catagen; T, telogen. Note the clustering of graph bars in groups corresponding to stages of synchronized hair cycling.

tomatically be limited to the hair follicular melanogenesis strictly coupled with the hair cycling. While in the cited paper (Plonka et al., 2005b) we focused mainly on telogen after a spontaneous hair cycle, here we have included more results from depilation-induced hair cycle, and our results are similar, as for the percentage of melanotic spleens (Table 1). Here we do not correlate melanosis with particular stages of the hair cycle; these results only serve as a positive control to show that splenic melanosis exists in young animals where the hair cycling is more or less synchronized (Dry, 1926; Chase, 1954).

We synchronized the hair cycle by depilation, but even in the spontaneous hair cycling in such young animals the hair cycle is synchronized (Dry, 1926), though not in the whole back skin. Waves of anagen are formed and transferred across the back skin according to well-defined patterns (Dry, 1926; Chase \& Eaton, 1959). 
Hair cycle de-synchronization and lack of macroscopic splenic melanosis in old C57BL/6 mice

Mice are rarely kept in laboratories for a long time, unless a long follow-up observation is necessary, which was the case for our long-term experiments done for other reasons. In Fig. 3 we show such an old mouse with spotty back skin being a mosaic of dark, black and pink areas devoid of, or covered with hair coat (Fig. 3A). The bald areas which emerge probably due to weaning are typical of C57BL/6 mice, and are described as "idiopatic dermatitis" in "a subpopulation" of this mouse strain (Sundberg \& King, 2000). Beside such, some old mice revealed macroscopic telogen, as judged by the pink colour of their back skin.

We observed spleens both in non-depilated and in depilated old animals (depilation, however, took place at least 2 years before spleen harvesting), in untreated and also in tested mice. In particular, we checked this phenomenon after a long-time treatment with MNA (Bryniarski et al., 2008), and after a single dose of CYP, in normal and Nos3 knockout mice. We decided to include these treated animals in this report because of the general shortage of old animals in professional animal facilities, and a lack of observations of such mice, and because of the perfect homogeneity of the results $(0 \%$ macroscopic melanosis).

The influence of pharmacological factors and nitric oxide on the splenic melanosis in young mice is a subject of different on-going experiments and their analysis would only complicate interpretation of the present study. However, we observed stronger or weaker splenic melanosis after treatment with MNA or CYP (or both), sometimes even stronger than in the control young animals (not shown). Consequently, we do not expect that the additional treatment might have negatively influenced our observations of the old mice. NOS 3 has not been reported to be engaged in the regulation of hair follicular pigmentation, but there are reports on such engagements of NOS 1, which in human cells may stimulate melanogenesis via protein kinase G (PKG) pathway (Roméro-Graillet et al., 1996; 1997; Slominski et al., 2004). Moreover, while knockout of Nos3 might theoretically decrease melanogenesis, the percentage of melanotic spleens in young $\mathrm{Nos}^{-/-}$animals is similar to that in wildtype C57BL/6 (Table 1). In a separate study we found that MNA does not affect follicular melanogenesis (not shown). Actually, we tried here to induce melanosis in old spleens using MNA (in vain - see Table 2). CYP transiently impairs follicular melanogenesis (Kostanecki et al., 1967; Paus et al., 1994), but this process is followed by regeneration of properly pigmented hair coat within two hair cycles following CYP administra- tion (Paus et al., 1994; Hendrix et al., 2005), while in our study CYP was administered once, 9 weeks after birth, i.e. over 2 years before observation. Not in a single mouse out of the observed animals $(n=37)$ coming from 12 groups was macroscopic splenic melanosis ever found (Fig. 3B, Table 2).

The mice included in the present experiments were carefully checked for de-synchronization of hair cycling, which manifests itself macroscopically (Fig. 3A). About $16 \pm 7 \%$ of back skin was of black colour indicating anagen/early catagen, while the rest $-85 \%$ was probably in telogen/late catagen/ early anagen. However, in the skin of old animals the pigmented spots might develop in a similar way as in humans, due to deposition of poorly degraded melanin or lipofuscin (Kent, 1976; Crichton et al., 1978; Goyarts et al., 2007). Therefore - a histological examination of skin was performed to show that, indeed, in old mice various hair follicles were in different stages of hair cycling.

Histological sections revealed irregular presence of hair follicles of all hair cycle stages in the investigated skin (Fig. 3C). Another indicator of de-synchronization of hair cycling in these animals is variable skin thickness (Fig. $3 C-$ arrowheads), which is highest during anagen VI, and lowest in telogen (Hansen et al., 1984; Plonka et al., 1995; 2005a; 2008 Mueller-Roever et al., 2001). In histomorphometric histograms particular hair cycle stages did not form any clearly distinguishable group (Fig. 3D). It must be emphasized that in old, spotty mice histomorphometry is strongly arbitrary, due to high irregularity of black spots present in skins of old animals (Militzer, 2001). If the histological samples were taken from other parts of skin, the resultant histomorphometry might look different. However, a synchronization of hair cycling, like that in young animals would never be observed. Even in the telogen skin of old animals, anagen may begin as randomly distributed round spots (Militzer, 2001) rather than a wave which spreads across the back skin. This gradual de-synchronization of hair cycling in ageing mice of the C57BL/6 genetic background has also been reported by other authors (Dry, 1926; Chase \& Eaton, 1959; Militzer, 2001; Young et al., 2003; Plikus \& Choung, 2004; 2008).

\section{Melanin and "melanin debris" in spleens of young and old mice, as revealed by FM staining}

While non-melanotic spleens of young animals almost never revealed histological melanin (Fig. 4A, D), black spots of melanotic spleens in young animals (Fig. 4B, E) examined using Fontana-Masson staining revealed clusters of melanotic tissue with, at least in part, extracellular localization of amorphous pigment (Fig. 4E). Sometimes these clusters 


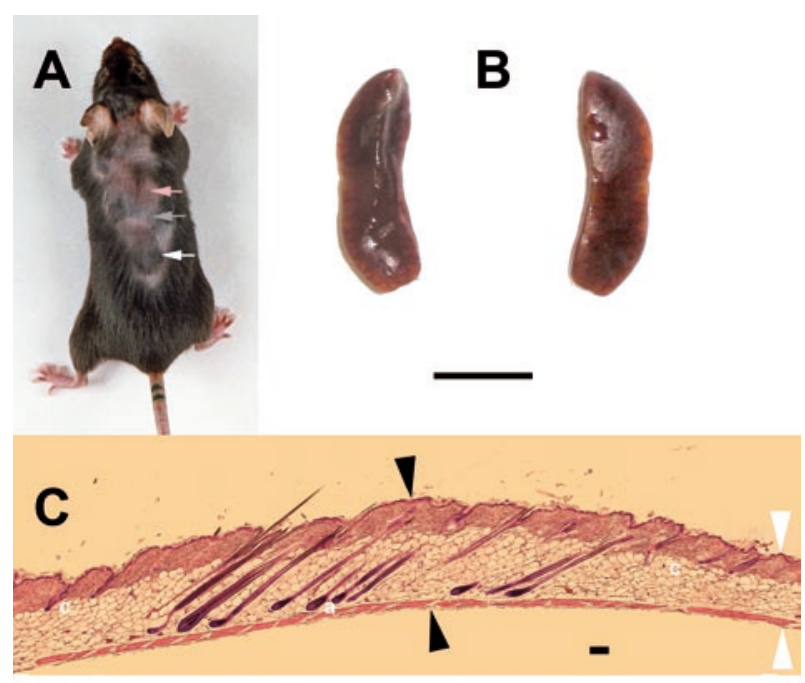

D

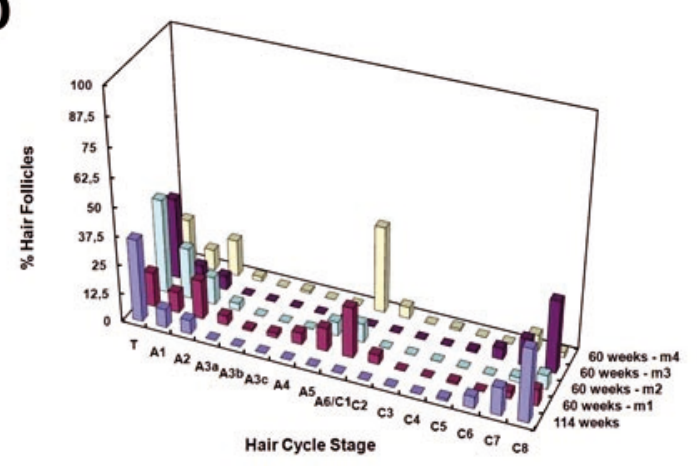

Figure 3. Association of a lack of macroscopic splenic melanosis in old C57BL/6 mice (60-133 weeks) with desynchronized hair cycling.

A, areas of variable pigmentation and baldness can be found in the back skin, which betray de-synchronization of hair cycling (white arrow - anagen, gray arrow - catagen, pink arrow - telogen region). B, spleens of such old C57BL/6 mice never reveal macroscopic melanosis (the dorsal and ventral region of the same spleen, scale bar $-5 \mathrm{~mm}$ ). C, histological skin section througt a melanotic spot ( $\mathrm{H} \& \mathrm{E}$ staining). Note the presence of hair follicles in distinct hair cycle stages (a, anagen; c, catagen). Arrowheads indicate differences in skin thickness between the anagen (black) and catagen region (white arrowheads). Scale bar, $0.1 \mathrm{~mm}$. D, histomorphometry of hair follicles in the middle part of the back skin of old mice (age in weeks and $\mathrm{m} 1-\mathrm{m} 4$, individual mice of 60-week group). A1-A6, C1-C8, T, stages (uppercase letters) and sub-stages (Arabic numbers and lowercase letters) of hair cycle. A, anagen; C, catagen; T, telogen. Note the dispersion of graph bars corresponding to particular stages of the hair cycle, which betrays its de-synchronization.

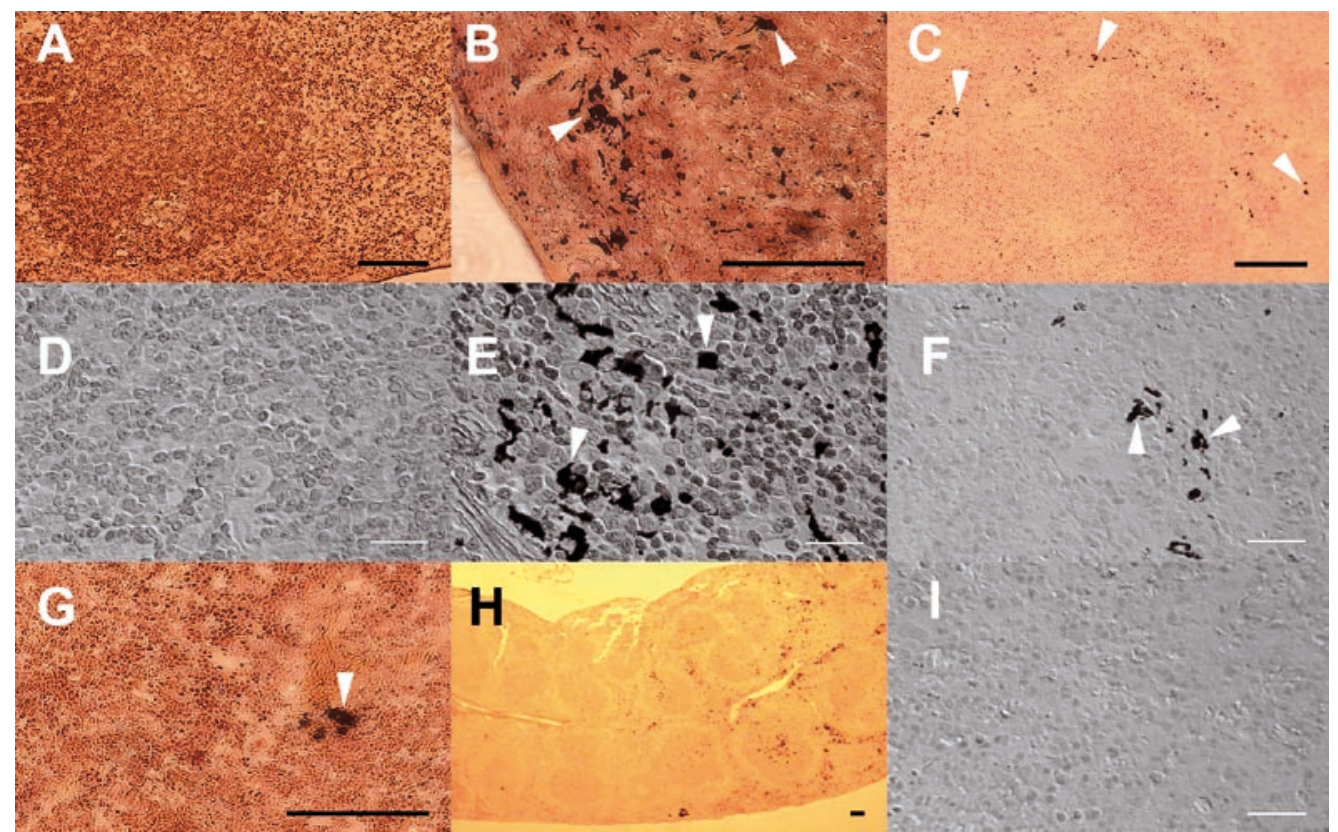

Figure 4. Histological examination of spleens of young and old C57BL/6 mice.

Fontana-Mason staining. A, young mouse, spleen without melanosis; B, young mouse, spleen with melanosis, the spot region; C, old mouse, fragment of spleen revealing melanin debris. D-F, corresponding pictures of A-C spleen sections, under large magnification in Nomarski interference contrast. G, bright region of a melanotic spleen (B) revealing melanin debris. $H$, the same spleen as (B) - lower magnification reveals the border between pigmented (on the right) and non-pigmented regions of the organ. I, spleen of old mouse $(\mathrm{C})$, section from region without melanin debris. Note extracellular localization of melanin (E, F). Arrowheads, melanin. Black scale bars $-0.1 \mathrm{~mm}$, white scale bars $-0.02 \mathrm{~mm}$. 
covered quite large areas of spleen tissue (Fig. 2B and $\mathrm{C}$ ). The border of the melanotic spot could also be found on histological sections (Fig $4 \mathrm{H}$ ), but no characteristic histological structures could be associated with such a transition region.

Interestingly, tiny FM-stainable particles were found in macroscopically non-melanotic spleens of old animals (Fig. 4C, F). However, not in every region (Fig. 4I), and only in about $40 \%$ of spleens. Such debris could also be found in spleens of young animals but only in the non-melanized parts of some melanotic spleens (Fig. 4G), not in normal non-melanotic spleens (Fig. 4A, D). Although the intrinsic ability to reduce silver (I) ions cannot be exclusively attributed to melanin granules and melanotic cells (Grimelius, 2008), both the localization and the intensity of staining did not differ from the FM-stained melanin depositions of the melanotic regions, therefore we are strongly convinced that the described particles represent melanin. We must, nevertheless, emphasize that it is very difficult to analyze the whole spleen in search of such "melanin debris", and it is quite probable that this phenomenon may be more frequent both in old and young animals.

\section{Cross-talk between synchronized hair cycling and splenic melanosis}

The phenomenon of splenic melanosis is related to the pigmentation phenotype and genotype of mice. If lipofuscins or products of non-enzymatic autooxidation and polymerization of degraded tissues were responsible, the phenomenon would also be present in albino mice. Wasserman (1967) and Crichton et al. (1978) examined various mouse strains and found no splenic melanosis in mice other than black. Crichton et al. (1978) report additionally splenic melanosis for inbread "Wr" and outbread "Q" strain. "Wr" mice are probably congenic wu/wu mice (Wobbler mice), where the wu (wobbler) mutation causing some neurological impairments can be introduced on various genetic backgrounds including C57BL/6 (Ishiyama et al., 1997). "Q mice" are probably randomly breeding outbread mice of various phenotypes (West \& McLaren, 1976). Unfortunately, Crichton et al. (1978) do not give any details concerning the pigmentation phenotypes of their laboratory animals. In a pilot study we did not find splenic melanosis in amelanotic mice but we did in some young agouti ones (not shown). This phenomenon needs, therefore, a thorough screening in the context of the popular genotypes and phenotypes of mice.

Only in young animals, whose hair cycle is synchronized, the melanotic spots can be found in spleens. Their appearance is partially correlated with the progress of the hair cycle (Plonka et al., 2005b), and they cannot be found in old animals with a desynchronized hair cycle. As ectopic melanin present, e.g., in the dermal papilla and in the outer root sheath is quite a frequent phenomenon in normal hair cycling (Tobin, 1998; Tobin et al., 1998), it may be the source for splenic melanin in young animals. Another source are apoptotic bodies from catagencoupled apoptosis of follicular melanocytes, which are initially phagocyted by Langerhans cells (Tobin 1998; Tobin et al., 1998; 1999). Their further fate remains unknown, but already in 1967 Wasserman suggested that there may be an alternate path of melanin transfer from skin and hair follicle melanocytes to the visceral organs with an engagement of phagocytic cells of the immune system (Wasserman, 1967). In aged mice, the mechanism of melanin scavenging may become imperfect, just like the general mechanisms of skin and hair pigmentation (Tobin \& Paus, 2001; Wulf et al., 2004). As a result - melanin cannot be transferred to other regions of the body and remains in the skin as melanotic spots (Kent, 1976; Goyarts et al., 2007). The de-synchronization of the hair cycle, however, may strongly decrease the total amount of melanin to be scavenged and deposited in the visceral organs. In both cases, hair follicles can be suggested as a source of splenic melanin, indicating the mutual dependence of both phenomena. If melanin were produced in spleens in situ, without any relation to the hair cycle (and the related melanogenesis) synchronization, it would be very difficult to explain why it is not visible in the spleens of old animals, in which anagen-coupled melanogenesis (Slominski \& Paus, 1993; Slominski et al., 1991; 1994; 2004; 2005) takes place, but is not synchronized over large areas of the skin.

\section{Melanin degradation in C57BL/6 animals}

The presence of "melanin debris" in nonpigmented spleens of old C57BL/6 animals may be a clue to answering the question about the fate of melanin in these spleens which do reveal melanosis in young age. The presence of "melanin debris" in old spleens is not a rule, but it appears in about $40 \%$ of animals, i.e. as frequently as does pigmentation of young spleens (Table 1). Melanin collected during subsequent hair cycles in spleens may be, therefore, gradually degraded to the "melanin debris", perhaps even more rapidly than the slow in-flow of new portions of melanin from de-synchronized hair follicles in catagen (Tobin et al., 1998, Tobin \& Paus, 2001).

Some authors question the actual nature of the splenic pigment. Hemosiderosis (Veninga et al., 1989 ) is a less probable reason of splenic pigmentation (Weissman, 1967; Sundberg, 1991; Van der Heijden et al., 1995). There is also controversy be- 
tween melanin and lipofuscin - pigments of different characters and origin (Kent, 1976; Double et al., 2008). Melanin is produced in melanosomes of melanocytes (Slominski et al., 2004; 2005), and lipofuscin - probably as a product of lipid oxidation and degradation by lysosomes (Crichton et al., 1978; Double et al., 2008). Here, however, the analogy between the development of melanosomes and autophagosomes should be mentioned (Schraermeyer, 1991; Palumbo, 2003), as well as the hypothesis on the origin of lipofuscins, at least in part (melanolipofuscin), from degraded melanosomes, including their lipid membrane structures, and even from melanin itself (Kayatz et al., 2001; Rozanowski et al., 2008). The process of melanosome degradation takes place both in the target keratinocytes (Borovansky \& Elleder, 2003; Slominski et al., 2004), and, probably, in melanophages (Langerhans cells), which partially degrade melanin phagocyted in catagen, and transfer it further to subsequent cells and visceral organs (Tobin, 1998; Tobin et al., 1998; 1999).

Melanin degradation is an important final step of the melanogenic pathway in mammals (Sulaimon \& Kitchell, 2003), which in human but not in mice appears primarily as degradation of melanosomes in epidermal keratinocytes (Borovansky \& Elleder, 2003; Slominski et al., 2004) removed subsequently from the organism together with the shed epidermis. Hair shaft melanin is probably not degraded, but disposed in toto in exogen - the shedding stage of the hair cycle (Tobin \& Paus, 2001; Milner et al., 2002; Slominski et al., 2004; Plonka et al., 2008). But, whatever is the real source of melanin in melanotic spleens of C57BL/6 mice, the pigment must be degraded there over the lifetime, as it is present in young and absent (or present only as "melanin debris") in old animals. If melanin were only stored in the spleens or synthesized de novo, but not degraded, the percentage of melanotic organs would increase over the lifetime, or remain on a stable level of at least $10-30 \%$.

The main way of melanin degradation is oxidative degradation by NADPH oxidases (Borovansky \& Elleder, 2003). The impact of this phenomenon on the viability of the organism may be considerable when it takes place in the internal compartments of the organism due to the potential toxicity of products of melanin and melanolipofuscin degradation (Hill \& Hill, 1987; Korytowski et al., 1987) and the possibility of releasing other substances "caged" in the structure of the polymer: toxins (e.g. drugs; Wilczok et al., 1990; Slominski et al., 2009) or metal ions (e.g. iron with its ability to drive Fenton reaction) (Sarna \& Plonka, 2005; Swartz et al., 2005; ShamotoNagai et al., 2006; Rozanowski et al., 2008).

Anyway, it is not possible to unambiguously state whether the "melanin debris" of the old ani- mals appears only in the "post-melanotic" spleens, or it can be present as the only manifestation of melanosis in some other, macroscopically non-melanotic spleens. Accordingly, another interpretation of our experiments is possible - one of the long-term experiments was performed initially on about 48 animals, and only 5 animals survived 133 weeks (Table 2), which makes about $10 \%$ of the initial number, and which is much less than the $30-40 \%$ of melanosis found in young animals. Their non-melanotic spleens may, therefore, not necessarily indicate that melanin is degraded over the mice lifetime, but instead that only animals without macroscopic splenic melanosis survive for so long.

\section{CONCLUSIONS}

Old C57BL/6 animals do not reveal macroscopic spleen melanosis, and do not reveal synchronized hair cycle, in contrast to young C57BL/6 mice. The mechanisms of hair cycle regulation (synchronization) appear to act in a systemic way, influencing the activity of various organs distant from the skin. Splenic melanosis, in the context of hair cycling in C57BL/6 mice, is an attractive and unique phenomenon to investigate mechanisms of ageing and melanin degradation in vivo.

\section{Acknowledgements}

We are very grateful to Professor Zbigniew Madeja from the Department of Cell Biology, Faculty of Biochemistry, Biophysics and Biotechnology Jagiellonian University (Kraków, Poland) for the possibility to use the Leica microscope with NIC, to Professor Stefan Chłopicki from the Department of Experimental Pharmacology, Chair of Pharmacology, Jagiellonian University Medical College (Kraków, Poland), for the spleens of Nos3 knockout mice, to the reviewers, and to the language editor.

This study was supported in part by grant PBZ-KBN-101/T09/2003/12 from the Ministry of Science and Informatization, Poland, to P.M.P.

\section{REFERENCES}

Bancroft JD, Stevens A (1982) Theory and practice of histological techniques. Churchill Livingstone, Edinburgh, Scotland.

Borovansky J, Elleder M (2003) Melanosome degradation: fact or fiction. Pigment Cell Res 16: 280-286.

Bryniarski K, Biedron R, Jakubowski A, Chlopicki S, Marcinkiewicz J (2008) Anti-inflammatory effect of 1-methylnicotinamide in contact hypersensitivity to oxazolone in mice; involvement of prostacyclin. Eur J Pharmacol 578: 332-338. 
Chase HB (1954) Growth of the hair. Physiol Rev 34: 113126.

Chase HB, Eaton GJ (1959) The growth of hair follicles in waves. Ann N Y Acad Sci 83: 365-368.

Crichton DN, Busuttil A, Price WH (1978) Splenic lipofuscinosis in mice. J Pathol 126: 113-120.

Double KL, Dedov VN, Fedorow H, Kettle E, Halliday GM, Garner B, Brunk UT (2008) The comparative biology of neuromelanin and lipofuscin in the human brain. Cell Mol Life Sci 65: 1669-1682.

Dry FW (1926) The coat of the mouse (Mus musculus). J Genet 16: 281-340.

Fontana A (1912) Verfahren zur intensiver und raschen Farbung des Treponema pallidum und anderer Spirochäten. Dermatol Wochenschr 55: 1003-1004 (in German).

Goyarts E, Muizzuddin N, Maes D, Giacomoni PU (2007) Morphological changes associated with aging: age spots and the microinflammatory model of skin aging. Ann N Y Acad Sci USA 1119: 32-39.

Grimelius L (2008) Methods in neuroendocrine histopathology. A methodological overview. Upsala J Med Sci 113: 243-260.

Hansen LS, Coggle JE, Wells J, Charles MW (1984) The influence of the hair cycle on the thickness of mouse skin. Anat Rec 210: 569-573.

Hendrix S, Handjiski B, Peters EM, Paus R (2005) A guide to assessing damage response pathways of the hair follicle: lessons from cyclophosphamide-induced alopecia in mice. J Invest Dermatol 125: 42-51.

Hill HZ, Hill GJ (1987) Eumelanin causes DNA strand breaks and kills cells. Pigment Cell Res 1: 163-170.

Ishiyama T, Klinkosz B, Pioro EP, Mitsumoto H (1997) Genetic transfer of the wobbler gene to a C57BL/6J $\times$ NZB hybrid stock: natural history of the motor neuron disease and response to CNTF and BDNF cotreatment. Exp Neurol 148: 247-255.

Izumi T, Gomi H, Kasai K, Mizutani S, Torii S (2003) The roles of Rab27 and its effectors in regulated secretory pathways. Cell Struct Funct 28: 465-474.

Kayatz P, Thumann G, Luther TT, Jordan JF, BartzSchmidt KU, Esser PJ, Schraermeyer U (2001) Oxidation causes melanin fluorescence. Invest Ophthalmol Vis Sci 42: 241-246.

Kent S (1976) Solving the riddle of lipofuscin's origin may uncover clues to the aging process. Geriatrics 31: 128137.

Korytowski W, Pilas B, Sarna T, Kalyanaraman B (1987) Photoinduced generation of hydrogen peroxide and hydroxyl radicals in melanin. Photochem Photobiol 45: 185-190.

Kostanecki W, Kwiatkowska E, Zak R (1967) The effect of Endoxan on hair melanogenesis and hair growth in mice. Arch Klin Exp Dermatol 230: 396-401 (in German).

Masson P (1914) La grande endocrine de l'intestin chez l'homme. CR Acad Sci (Paris) 138: 59-61 (in French).

Militzer K (2001) Hair growth pattern in nude mice. Cells Tissues Organs 168: 285-294.

Milner Y, Sudnik J, Filippi M, Kizoulis M, Kashgarian M, Stenn K (2002) Exogen, shedding phase of the hair growth cycle: Characterization of a mouse model. J Invest Dermatol 119: 639-644.

Mueller-Roever S, Handjiski B, van der Veen C, Eichmuller S, Foitzik K, McKay IA, Stenn KS, Paus R (2001) A comprehensive guide for the accurate classification of murine hair follicles in distinct hair cycle stages. J Invest Dermatol 117: 3-15.

Palumbo A (2003) Melanogenesis in the ink gland of Sepia officinalis. Pigment Cell Res: 16: 517-522.
Paus R, Christoph T, Müller-Röver S (1999) Immunology of the hair follicle: a short journey into terra incognita. J Invest Dermatol Symp Proc 4: 226-234.

Paus R, Handjiski B, Eichmüller S, Czarnetzki BM (1994) Chemotherapy-induced alopecia in mice. Induction by cyclophosphamide, inhibition by cyclosporine A, and modulation by dexamethasone. Am J Pathol 144: 719734.

Paus R, Stenn KS, Link RE (1990) Telogen skin contains an inhibitor of hair growth. Br J Dermatol 122: 777-784.

Plikus MV, Chuong CM (2008) Complex hair cycle domain patterns and regenerative hair waves in living rodents. J Invest Dermatol 128: 1071-1080.

Plikus M, Chuong CM (2004) Making waves with hairs. J Invest Dermatol 122: vii-ix.

Plonka P, Plonka B, Paus R (1995) Biophysical monitoring of melanogenesis as a tool for pigment and hair research. Arch Dermatol Res 287: 687-690.

Plonka PM, Handjiski B, Popik M, Michalczyk D, Paus R (2005a) Zinc as an ambivalent but potent modulator of murine hair growth in vivo - preliminary observations. Exp Dermatol 14: 844-853.

Plonka PM, Michalczyk D, Popik M, Handjiski B, Slominski A, Paus R (2005b) Splenic eumelanin differs from hair eumelanin in C57BL/6 mice. Acta Biochim Polon 52: 433-441.

Plonka PM, Michalczyk D, Popik M, Handjiski B, Paus R (2008) Electron paramagnetic resonance (EPR) spectroscopy for investigating murine telogen skin after a spontaneous or depilation-induced hair growth. J Dermatol Sci 49: 227-240.

Romeis B (1991) Mikroskopische Technik. Urban \& Schwarzenberg, Muenchen, Germany (in German).

Roméro-Graillet C, Aberdam E, Aberdam E, Biagoli N, Ortonne J-P, Ballotti R (1996) Ultraviolet B radiation acts through the nitric oxide and cGMP signal transduction pathway to stimulate melanogenesis in human melanocytes. J Biol Chem 271: 28052-28056.

Roméro-Graillet C, Aberdam E, Clément M, Ortonne J-P, Ballotti R (1997) Nitric oxide produced by ultravioletirradiated keratinocytes stimulates melanogenesis. J Clin Invest 99: 635-642.

Rozanowski B, Cuenco J, Davies S, Shamsi FA, Zadlo A, Dayhaw-Barker P, Rozanowska M, Sarna T, Boulton ME (2008) The phototoxicity of aged human retinal melanosomes. Photochem Photobiol 84: 650-657.

Sarna T, Plonka PM (2005) Biophysical studies of melanin: paramagnetic, ion-exchange and redox properties of melanin pigments and their photoreactivity. In Biomedical ESR. Biological Magnetic Resonance Series. Eaton SS, Eaton GR, Berliner LJ, eds, vol 23, pp 125-146. Kluwer Academic Publishers, The Netherlands, New York, Boston.

Schneider MR, Schmidt-Ullrich R, Paus R (2009) The hair follicle as a dynamic miniorgan. Curr Biol 19: R132R142.

Schraermeyer U (1991) Fine structure of melanogenesis in the ink sack of Sepia officinalis. Pigment Cell Res 7: 52-60.

Shamoto-Nagai M, Maruyama W, Yi H, Akao Y, Tribl F, Gerlach M, Osawa T, Riederer P, Naoi M (2006) Neuromelanin induces oxidative stress in mitochondria through release of iron: mechanism behind the inhibition of 26 S proteasome. J Neural Transm 113: 633-644.

Shirai A, Tsunoda H, Tamaoki T, Kamiya T (2001) Topical application of cyclosporine A induces rapid-remodeling of damaged anagen hair follicles produced in cyclophosphamide administered mice. J Dermatol Sci 27: 7-13. 
Slominski A, Paus R (1993) Melanogenesis is coupled to murine anagen: toward new concepts for the role of melanocytes and the regulation of melanogenesis in hair growth. I Invest Dermatol 101: 90S-97S.

Slominski A, Paus R, Costantino R (1991) Differential expression and activity of melanogenesis-related proteins during induced hair growth in mice. J Invest Dermatol 96: 172-179.

Slominski A, Paus R, Plonka P, Maurer M, Chakraborty A, Pruski D, Lukiewicz S (1994) Melanogenesis during the anagencatagen-telogen transformation of the murine hair cycle. J Invest Dermatol 102: 862-869.

Slominski A, Goodman-Snitkoff G, Maurer M, Paus R (1997) Hair cycle-associated changes in splenocyte proliferation. In Vivo 11: 101-102.

Slominski A, Tobin DJ, Shibahara S, Wortsman J (2004) Melanin pigmentation in mammalian skin and its hormonal regulation. Physiol Rev 84: 1155-1228.

Slominski A, Wortsman J, Plonka PM, Schallreuter KU, Paus R, Tobin DJ (2005) Hair follicle pigmentation. J Invest Dermatol 124: 13-21.

Slominski A, Zbytek B, Slominski R (2009) Inhibitors of melanogenesis increase toxicity of cyclophosphamide and lymphocytes against melanoma cells. Int J Cancer 124: 1470-1477.

Stenn KS, Paus R (2001) Controls of hair follicle cycling. Physiol Rev 81: 449-494.

Sulaimon SS, Kitchell BE (2003) The biology of melanocytes. Vet Dermatol 14: 57-65.

Sundberg JP (1991) Pigmented spleens in C57BL mice. Labor Anim 25: 85-86.

Sundberg JP, King LE Jr (2000) Skin and its appendages: Normal anatomy and pathology of spontaneous, transgenic, and targeted mouse mutations. In Pathology of genetically engineered mice. Ward JM, Mahler JF, Marnopt RR, Sundberg JP, Frederickson RM, eds, pp 183215. Iowa State University Press, Ames, IA.

Swartz HM, Mason RP, Hogg N, Kalyanaraman B, Sarna T, Plonka PM, Zareba M, Gutierrez PL, Berliner LJ (2005) Free radicals and medicine. In Biomedical ESR. Biological Magnetic Resonance Series. Eaton SS, Eaton GR, Berliner LJ, eds, vol. 23, pp 25-74. Kluwer Academic Publishers, The Netherlands, New York, Boston.
Tobin DJ (1998) A possible role for Langerhans cells in the removal of melanin from early catagen hair follicle. $\mathrm{Br}$ J Dermatol 168: 795-798.

Tobin D J, Paus R (2001) Graying: Gerontobiology of the hair follicle pigmentary unit. Exp Gerontol 36: 29-54.

Tobin DJ, Hagen E, Botchkarev VA, Paus R (1998) Do hair bulb melanocytes undergo apoptosis during hair follicle regression (catagen)? J Invest Dermatol 111: 941-947.

Tobin DJ, Slominski A, Botchkarev V, Paus R (1999) The fate of hair follicle melanocytes during the hair growth cycle. J Invest Dermatol Symp Proc 4: 323-332.

Van den Bossche K, Naeyaert J-M, Lambert J (2006) The quest for the mechanism of melanin transfer. Traffic 7 : 769-778.

Van der Heijden A, van Dijk JE, Lemmens AG, Beynen AC (1995) Spleen pigmentation in young C57BL mice is caused by accumulation of melanin. Labor Anim 29: 459-463.

Veninga TS, Wieringa RA, Morse H (1989) Pigmented spleens in C57BL mice. Labor Anim 23: 16-20.

Wassermann HP (1967) Extension of the concept "vertebrate epidermal melanin unit" to embrace visceral pigmentation and leucocytic melanin transport. Nature 213: 282-283.

Weissman I (1967) Genetic and histochemical studies on mouse spleen black spots. Nature 215: 315.

West JD, McLaren A (1976) The distribution of melanocytes in the dorsal coats of a series of chimaeric mice. $J$ Embryol Exp Morphol 35: 87-93.

Wilczok T, Stepień K, Buszman E, Porebska-Budny M (1990) Interaction of methotrexate with melanins and melanosomes from B16 melanoma. Biophys Chem 35: 265-270.

Wulf HC, Sandby-Møller J, Kobayasi T, Gniadecki R (2004) Skin aging and natural photoprotection. Micron 35: 185-191.

Young P, Boussadia O, Halfter H, Grose R, Berger P, Leone DP, Robenek H, Charnay P, Kemler R, Suter U (2003) E-cadherin controls adherens junctions in the epidermis and the renewal of hair follicles. $E M B O J \mathbf{2 2}$ : 5723-5733. 